Article

\title{
Direct Synthesis of Phosphonates and $\alpha$-Amino-phosphonates from 1,3-Benzoxazines
}

\author{
Oscar Salgado-Escobar ${ }^{1}$, Alexis Hernández-Guadarrama ${ }^{1}$, Ivan Romero-Estudillo ${ }^{2}$ and \\ Irma Linzaga-Elizalde ${ }^{1, *}$
}

1 Centro de Investigaciones Químicas-IICBA, Universidad Autónoma del Estado de Morelos, Av. Universidad 1001, 62209 Cuernavaca, Morelos, México; pyrel@hotmail.com (O.S.-E.); alexis13975@hotmail.com (A.H.-G.)

2 CONACYT-Centro de Investigaciones Químicas-IICBA, Universidad Autónoma del Estado de Morelos, Av. Universidad 1001, 62209 Cuernavaca, Morelos, México; ivan.romeroest@uaem.mx

* Correspondence: linzaga@uaem.mx; Tel.: +52-777-329-7997

Received: 22 November 2018; Accepted: 11 January 2019; Published: 15 January 2019

\begin{abstract}
A straightforward and novel method for transformation of readily available1,3-benzoxazines to secondary phosphonates and $\alpha$-aminophosphonates using boron trifluoride etherate as catalyst is developed. The formation of phosphonates proceeds through ortho-quinone methide (o-QM) generated in situ, followed by a phospha-Michael addition reaction. On the other hand, the $\alpha$-aminophosphonates were obtained by iminium ion formation and the subsequence nucleophilic substitution of alkylphosphites. This method can be also used for the preparation of $o$-hydroxybenzyl ethers through oxa-Michael addition.
\end{abstract}

Keywords: phosphonates; $\alpha$-aminophosphonates; $o$-quinone methide; $o$-hydroxybenzylic ethers; 1,3-benzoxazines

\section{Introduction}

The $\alpha$-aminophosphonic acids are probably the most important analogues of $\alpha$-amino acids attributed to their structural analogy obtained by isosteric substitution of planar carboxylic acid $\left(\mathrm{CO}_{2} \mathrm{H}\right)$ by tetrahedral phosphonic acid $\left(\mathrm{PO}_{3} \mathrm{H}_{2}\right)$ [1-3]. This kind of compounds have been widely studied and used in agriculture, industry and medicinal chemistry [4-9]. In this context, the phosphonates and $\alpha$-aminophosphonates also constitute an interesting class of compounds which have been utilized in the production of dental additives [10,11], dispersants, corrosion inhibitors [12-14], in fire retardants [15-17], as well as for preventing deposit formation [18]. Due the different applications, several efforts have been developed for the preparation of phosphonates and $\alpha$-aminophosphonates $[19,20]$.

In general, the main strategy for the synthesis of phosphonates including the Michaelis-Arbuzov [21] and Michaelis-Becker [22] reactions. On the other hand, the $\alpha$-aminophosphonates are commonly prepared by Kabachnik-Fields [23-25] or Pudovik reactions [26,27]. In this context, Chen [28] and Huang [29] described a practical method for the preparation of ortho-hydroxybenzyl phosphonates by phospha-Michael addition of phosphites to ortho-quinone methides (o-QMs). Particularly, the $o$-hydroxybenzyl phosphonates have been used for the preparation of 1,2-benzoxaphospholes with interesting antioxidants properties [30,31] and as anticancer agents [32].

Considering the high value of these compounds and in connection with our recent work [33], we report herein an innovative methodology for the synthesis of secondary phosphonates and $\alpha$-aminophosphonates from the reaction of 1,3-benzoxazines with diethyl or triethyl phosphite using catalytic amounts of boron trifluoride etherate. In addition, when the 1,3-benzoxazines was treated with alcohols under reflux conditions provided the corresponding ethers in good yields. 


\section{Results and Discussion}

Initially, 1,3-benzoxazines $\mathbf{1 a - h}$ were prepared from the corresponding 2-(benzylamino)phenols following procedures described in the literature [34-36]. In the next step, the study of the reaction conditions for the synthesis of the phosphonate $\mathbf{2 b}$ and $\alpha$-aminophosphonate $\mathbf{3 b}$ were started. For this purpose, the reaction of the 1,3-benzoxazine $\mathbf{1} \mathbf{b}$ and triethyl phosphite under different conditions (solvents, temperature and using boron trifluoride etherate as catalyst) was examined in order to find the best reaction conditions (Table 1). At first, the 1,3-benzoxazine $\mathbf{1 b}$ was treated with triethyl phosphite in ethanol obtaining the $\alpha$-aminophosphonate $3 \mathbf{b}$ in $28 \%$ yield (Table 1 , entry 1 ). In entry 2 was carried out the reaction at $26^{\circ} \mathrm{C}$ in presence of catalytic amounts of boron trifluoride etherate (20 $\mathrm{mol} \%$ ) using DCM as solvent afforded the $\alpha$-aminophosphonate $3 \mathbf{b}$ in $27 \%$ yield. On the other hand, using the same solvent at $40{ }^{\circ} \mathrm{C}$ and without catalysts the result was similar ( $3 \mathrm{~b} ; 28 \%$ yield, entry 3). In the next experiments, using $\mathrm{MeCN}$ as solvent at 26 and $82{ }^{\circ} \mathrm{C}$ without catalyst, product reaction was not formed (entries 4 and 5).

Alternatively, when MeCN was used in presence of catalytic amounts of boron trifluoride etherate $(10 \mathrm{~mol} \%)$ at $26^{\circ} \mathrm{C}$ the phosphonate $\mathbf{2 b}$ in $18 \%$ yield was afforded (entry 6$)$. On the other hand, from the reaction of the 1,3-benzoxazine $\mathbf{1} \mathbf{b}$ with triethyl phosphite and increasing amount of boron trifluoride etherate at 20 and $50 \mathrm{~mol} \%, \mathbf{2 b}$ in $28 \%$ yield was obtained in both cases (entries 7 and 8 ). Then 2.7 equivalents of triethyl phosphite were used and the phosphonate $\mathbf{2 b}$ was isolated in $28 \%$ yield (entry 9). In entry 10 the reaction mixture was refluxed in $\mathrm{MeCN}$ with the presence of boron trifluoride etherate $(20 \mathrm{~mol} \%)$, from these, the phosphonate $\mathbf{2 b}$ and $\alpha$-aminophosphonate $\mathbf{3 b}$ in 30 and $47 \%$ yield respectively were afforded.

Table 1. Study of the reaction of 1,3-benzoxazine $\mathbf{1 b}$ with triethyl phosphite.

\begin{tabular}{|c|c|c|c|c|c|}
\hline \multicolumn{2}{|c|}{$1 \mathrm{~b}$} & $\frac{\mathrm{Et})_{3}, \mathrm{BF}_{3} \cdot \mathrm{OEt}}{\text { solvent, } 72 \mathrm{~h}}$ & $2 b$ & $3 \mathbf{b}$ & $\begin{array}{l}\mathrm{O} \\
\mathrm{P}(\mathrm{OEt})_{2}\end{array}$ \\
\hline Entry & Solvent & Temp. $\left({ }^{\circ} \mathrm{C}\right)$ & $\mathrm{P}(\mathrm{OEt})_{3}(\mathrm{Eq})$ & $\mathrm{BF}_{3} \mathrm{OEt}_{2}(\mathrm{~mol} \%)$ & Yield (\%) \\
\hline 1 & $\mathrm{EtOH}$ & 78 & 1.0 & - & $3 b ; 28$ \\
\hline 2 & DCM & 26 & 1.0 & 20 & $3 b ; 27$ \\
\hline 3 & DCM & 40 & 1.5 & - & $3 b ; 28$ \\
\hline 4 & $\mathrm{MeCN}$ & 26 & 1.0 & - & No product \\
\hline 5 & $\mathrm{MeCN}$ & 82 & 1.0 & - & No product \\
\hline 6 & $\mathrm{MeCN}$ & 26 & 2.0 & 10 & $2 b ; 18$ \\
\hline 7 & $\mathrm{MeCN}$ & 26 & 2.1 & 20 & $2 \mathbf{b} ; 28$ \\
\hline 8 & $\mathrm{MeCN}$ & 26 & 2.2 & 50 & $2 b ; 28$ \\
\hline 9 & $\mathrm{MeCN}$ & 26 & 2.7 & 50 & $2 b ; 28$ \\
\hline 10 & $\mathrm{MeCN}$ & 82 & 3.0 & 20 & $\mathbf{2 b} ; 30,3 \mathbf{b} ; 47$ \\
\hline 11 & $\mathrm{MeCN}$ & 82 & 3.5 & 20 & $\mathbf{2 b} ; 28,3 \mathbf{b} ; 47$ \\
\hline 12 & Hexane & 26 & 3.6 & 20 & No product \\
\hline
\end{tabular}

In another experiment, an increase to 3.5 equivalents of the triethyl phosphite under similar conditions did not improve the yield (entry 11). When hexane was used as solvent, no products were observed (Table 1 , entry 12).

With these results the formation of the phosphonate was favored when triethyl phosphite, boron trifluoride etherate in catalytic quantities and a polar solvent as acetonitrile at room temperature were used, besides, the reaction was cleaner and the 1,3-benzoxazine that not reacted was recovered.

Under the optimized conditions, the 1,3-benzoxazines $\mathbf{1 a}-\mathbf{h}$ were reacted with triethyl phosphite in presence of boron trifluoride etherate $(20 \mathrm{~mol} \%)$ in acetonitrile (Scheme 1). When the 1,3-benzoxazines $\mathbf{1 b}, \mathbf{1 e}$ and $\mathbf{1 g}$ were used, the $o$-hydroxybenzyl phosphonates $\mathbf{2 b}, \mathbf{2 e}$ and $\mathbf{2} \mathbf{g}$ were formed in $28-40 \%$ 
yields. The $o$-hydroxybenzyl phosphonates are valuable building block for the synthesis of a wide range of compounds. [29,30,37,38]. From 1,3-benzoxazines 1a, $\mathbf{1 d}$ and $\mathbf{1 h}$ the $\alpha$ - amino-phosphonates 3a, 3d and $\mathbf{3 h}$ were obtained in 6-89\% yields (Scheme 1).

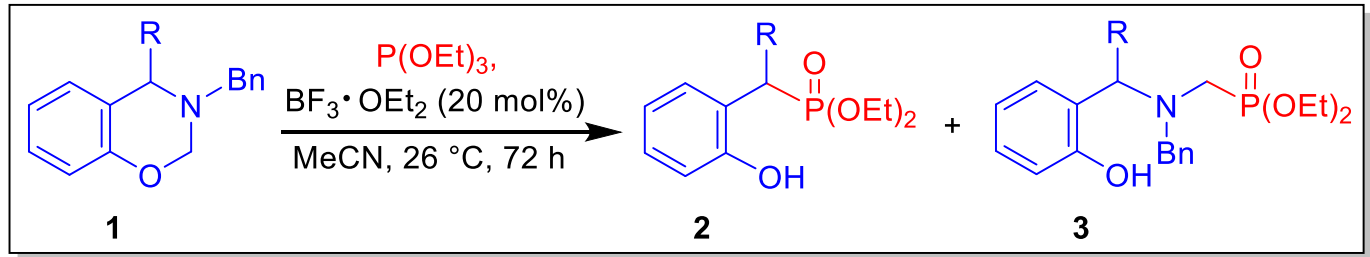

$\begin{array}{llllll}\text { 1a; } \mathrm{R}=\mathrm{H} & \mathbf{1 e} ; \mathrm{R}=\mathrm{C}_{6} \mathrm{H}_{5} & \mathbf{2 a} ; 0 \% & \mathbf{2 e} ; 40 \% & \mathbf{3 a} ; 89 \% & \mathbf{3 e} ; 0 \% \\ \mathbf{1 b} ; \mathrm{R}=\mathrm{Me} & \mathbf{1 f} ; \mathrm{R}=m-\mathrm{MeC}_{6} \mathrm{H}_{4} & \mathbf{2 b} ; \mathbf{2 8 \%} & \mathbf{2 f} ; 0 \% & \mathbf{3 b} ; 0 \% & \mathbf{3 f} ; 0 \% \\ \mathbf{1 c} ; \mathrm{R}=n-\mathrm{Bu} & \mathbf{1 g} ; \mathrm{R}=p-\mathrm{MeC}_{6} \mathrm{H}_{4} & \mathbf{2 c} ; 0 \% & \mathbf{2 g} ; 30 \% & \mathbf{3 c} ; 0 \% & \mathbf{3 g} ; 0 \% \\ \text { 1d; } \mathrm{R}=s-\mathrm{Bu} & \mathbf{1 h} ; \mathrm{R}=p-\mathrm{ClC}_{6} \mathrm{H}_{4} & \mathbf{2 d} ; 0 \% & \mathbf{2 h} ; 0 \% & \mathbf{3 d} ; \mathbf{3 7 \%} & \mathbf{3 h} ; 6 \%\end{array}$

Scheme 1. Synthesis of phosphonates and $\alpha$-aminophosphonates from 1,3-benzoxazines.

The mechanism in Scheme 4 below shows an equilibrium in the ring-opening benzoxazines via iminium ion or $o$-Quinone Methide (o-QMs) intermediates. Considering that the stabilization of the iminium ions is directly affected by the steric effect of the substituent $\left(\mathrm{H}>\mathrm{Me}>n\right.$ - $\mathrm{Bu}>s-\mathrm{Bu}>\mathrm{C}_{6} \mathrm{H}_{5} \geq$ $\left.p-\mathrm{ClC}_{6} \mathrm{H}_{5}>p-\mathrm{MeC}_{6} \mathrm{H}_{4}>m-\mathrm{MeC}_{6} \mathrm{H}_{4}\right)$, the aminophosphonates with $\mathrm{H}$ and $\mathrm{Me}$ as substituents were formed in better yields. On the other side, the phosphonates were formed according to the stabilization of the substituent in $o$-QMs intermediates $\left(\mathrm{H}>\mathrm{Me}>n\right.$ - $\mathrm{Bu}>s-\mathrm{Bu}>\mathrm{C}_{6} \mathrm{H}_{5}>p-\mathrm{ClC}_{6} \mathrm{H}_{4}>p-\mathrm{MeC}_{6} \mathrm{H}_{4}>$ $\left.m-\mathrm{MeC}_{6} \mathrm{H}_{4}\right)$.

In order to study others phosphorus sources, the reaction of the 1,3-benzoxazines $\mathbf{1 a}-\mathbf{h}$, diethyl phosphite and boron trifluoride etherate as catalyst in MeCN were carried out (Scheme 2). To our satisfaction only the $\alpha$-aminophosphonates $3 \mathbf{a}-\mathbf{h}$ were detected in $24-96 \%$ yield. We found that the 1,3-benzoxazines $\mathbf{1 a}$ and $\mathbf{1 b}$ with hydrogen and methyl substituents show the best yields (96 and $80 \%$, respectively), whereas, the 1,3-benzoxazines $\mathbf{1 d}, \mathbf{1 g}$ and $\mathbf{1 h}$ with bulky substituents furnished the $\alpha$-aminophosphonates $\mathbf{3 d}, \mathbf{3 g}$ and $\mathbf{3 h}$ in moderate yields (Scheme 2). Due to the fact benzyl and $o$-hydroxylbenzyl groups are attached to the nitrogen atom, both move away from each other avoiding the steric hindrance, which causes them to be oriented towards the double bond of the iminium ion inhibiting the access of the phosphite. However, the 1,3-benzoxazine ring opening produces the reaction between the phenolate and hydrogen atom of diethyl phosphite tautomer $\left(\mathrm{Ar}-\mathrm{O}^{-}-\mathrm{H}-\mathrm{O}-\mathrm{P}\right)$, this facilitate the attack to form the C-P bond, this effect does not occur when triethyl phosphite is used.

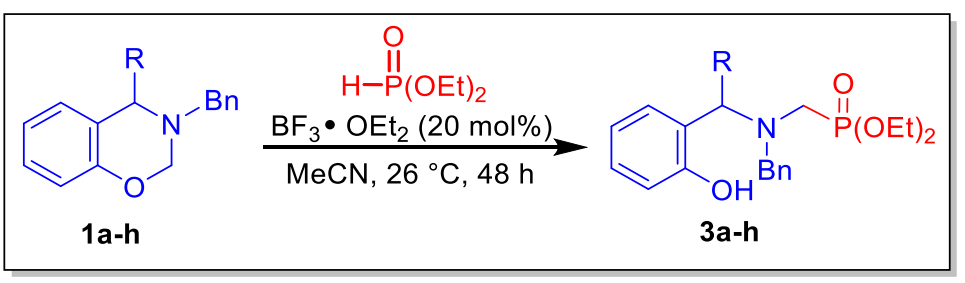

3a; $\mathrm{R}=\mathrm{H} ; 96 \%$

3b; $\mathrm{R}=\mathrm{Me} ; 80 \%$

3c; $\mathrm{R}=n-\mathrm{Bu} ; 58 \%$

3d; $\mathrm{R}=\mathrm{s}-\mathrm{Bu} ; 37 \%$

$3 e ; \mathrm{R}=\mathrm{C}_{6} \mathrm{H}_{5} ; 65 \%$

3f; $\mathrm{R}=m-\mathrm{MeC}_{6} \mathrm{H}_{4} ; 35 \%$

$3 \mathrm{~g} ; \mathrm{R}=p-\mathrm{MeC}_{6} \mathrm{H}_{4} ; 24 \%$

3h; $\mathrm{R}=p-\mathrm{ClC}_{6} \mathrm{H}_{4} ; 50 \%$

Scheme 2. Direct conversion of 1,3-benzoxazines 1a-h to $\alpha$-aminophosphonates 3a-h.

With the results obtained in the phosphorylation of $o-\mathrm{QMs}$, next we explored the direct transformation of 1,3-benzoxazine 1e. Thus, 1e was treated with 3-chloro-1-propanol at $70{ }^{\circ} \mathrm{C}$ for $12 \mathrm{~h}$ affording the oxa-Michael adduct $5 \mathrm{a}$ in $53 \%$ yield. The ether product is a versatile intermediate to obtain more complex compounds [29,39-41] (Scheme 3). 


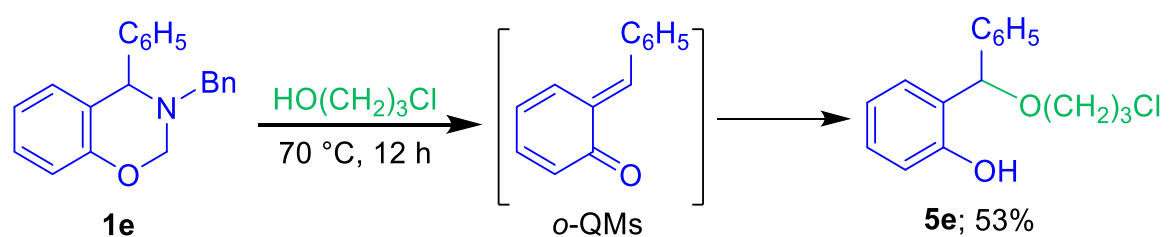

Scheme 3. Preparation of phenol ether 5e from the direct transformation of the 1,3-benzoxazine 1e.

A proposed reaction pathway is depicted in Scheme 4. The formation of $\alpha$-aminophosphonates can be explained through protonation of the oxygen by the hydrogen of diethyl phosphite which promotes the ring-opening generating the iminium ion, the subsequent phosphorylation provides the corresponding $\alpha$-aminophosphonates. On the other hand, when triethyl phosphite is used the electronic delocalization of electron pair of nitrogen could generate the ring opening of 1,3-benzoxazines producing the iminium ion (path A) [42,43] which is attacked by the triethyl phosphite to give the $\alpha$-aminophosphonates. When the oxygen was activated (path B) it promoted $o$-QM formation following by phospha-Michael addition reaction [28] with $\mathrm{P}(\mathrm{OEt})_{3}$ to produce the corresponding phosphonates.

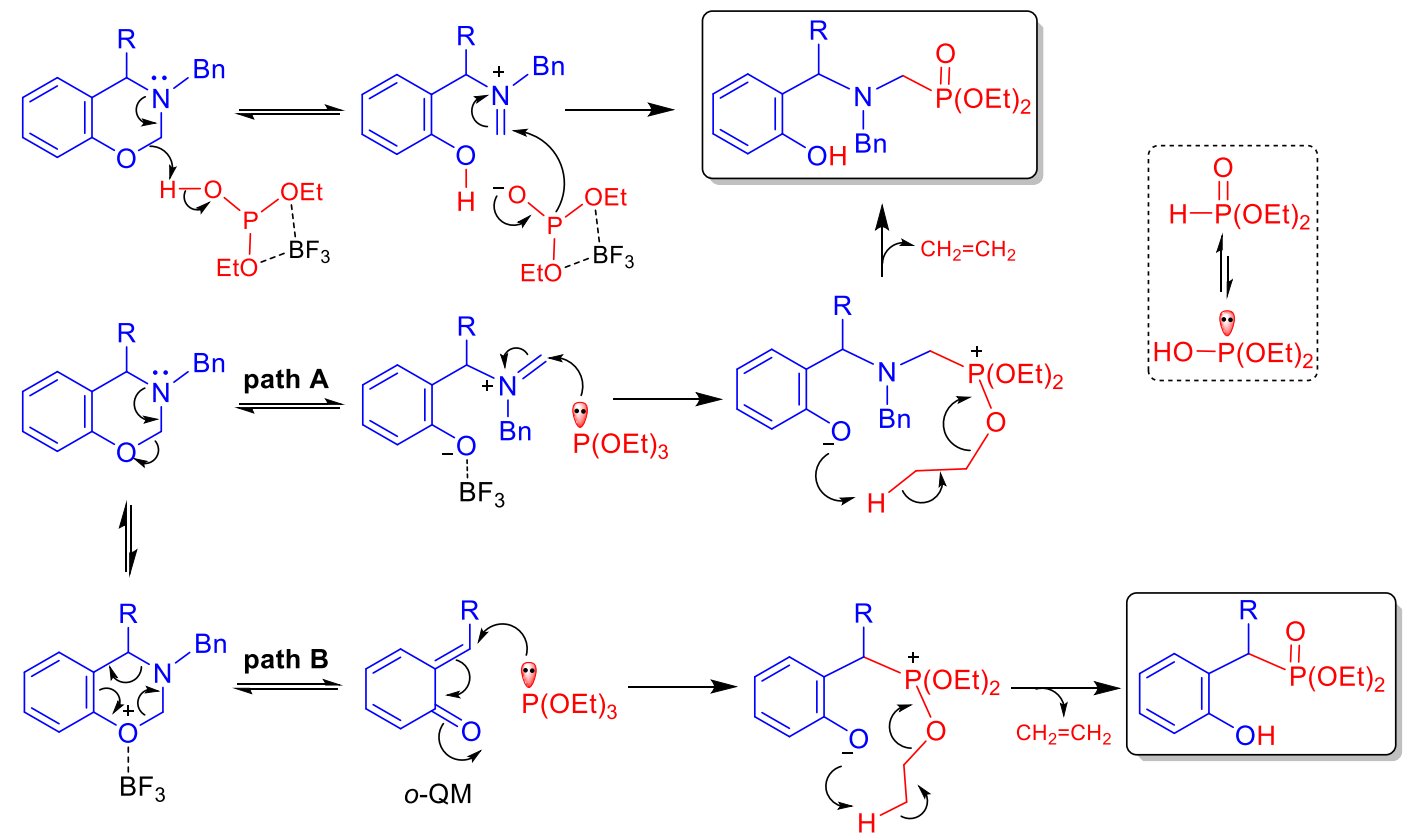

Scheme 4. Proposed reaction pathway for the ring-opening of 1,3-benzoxazines to generated phosphonates and $\alpha$-aminophosphonates.

\section{Materials and Methods}

\subsection{General Information}

Reagents were obtained from commercial suppliers and were used without further purifification. Melting points were determined in a Fischer Johns apparatus (Pittsburgh, PA, USA) and are uncorrected. NMR spectra were recorded on Varian System instrument (Palo Alto, CA, USA) at $400 \mathrm{MHz}$ for ${ }^{1} \mathrm{H}-$ and $100 \mathrm{MHz}$ for ${ }^{13} \mathrm{C}$ - and a Varian Gemini at $200 \mathrm{MHz}$ for ${ }^{1} \mathrm{H}$ - and $50 \mathrm{MHz}$ for ${ }^{13} \mathrm{C}$-. The spectra were obtained in $\mathrm{CDCl}_{3}$ solutions using TMS as an internal reference. ${ }^{31} \mathrm{P}$ chemical shifts are reported relative to $\mathrm{H}_{3} \mathrm{PO}_{4}$ as an internal reference. High-resolution $\mathrm{CI}^{+}$and $\mathrm{FAB}^{+}$mass experiments were performed on a JEOL HRMStation JHRMS-700 (Akishima, Tokyo, Japan). The purifification of all compounds was carried out by column chromatography using (silica gel 230-400 mesh). The dichloromethane and acetonitrile were reflfluxed on phosphorous pentoxide and hexane with sodium and benzophenone. Formaldehyde (30\%) was used for the reactions. 


\subsection{General Procedure to Obtain the 1,3-benzoxazines $1 a-h$}

A mixture of 2-(benzylamino)-phenol (1.0 eq.) and formaldehyde solution (1.3 eq.) in dichloromethane was stirred at $37^{\circ} \mathrm{C}$ for $1 \mathrm{~h}$ using a modified Dean-Stark tramp. The crude product was purified by flash chromatography using hexane:EtOAc (99:01) or by recrystallization in methanol.

\subsubsection{3-Benzyl-3,4-dihydro-2H-1,3-benzoxazine (1a)}

The ${ }^{1} \mathrm{H}$ - and ${ }^{13} \mathrm{C}-\mathrm{NMR}$ data for the compound $1 \mathrm{a}$ were identical to those reported in the literature [36].

\subsubsection{3-Benzyl-4-methyl-3,4-dihydro-2H-1,3-benzoxazine (1b)}

According to the general procedure, a mixture of 2-\{1-(benzylamino)ethyl\}phenol (1.0 g, $4.40 \mathrm{mmol})$ and formaldehyde $(0.17 \mathrm{~g}, 5.72 \mathrm{mmol}, 0.46 \mathrm{~mL})$ in dichloromethane $(10 \mathrm{~mL})$ was reacted. After purification, $\mathbf{1 b}(1.01 \mathrm{~g}, 99 \%)$ was obtained as a colorless oil. ${ }^{1} \mathrm{H}-\mathrm{NMR}\left(\mathrm{CDCl}_{3}, 400 \mathrm{MHz}\right)$ : $\delta 1.46(\mathrm{~d}, J=7.2 \mathrm{~Hz}, 3 \mathrm{H}), 3.74(\mathrm{q}, J=6.4 \mathrm{~Hz}, 1 \mathrm{H}), 3.79(\mathrm{~d}, J=13.6 \mathrm{~Hz}, 1 \mathrm{H}), 4.01(\mathrm{~d}, J=14.0 \mathrm{~Hz}, 1 \mathrm{H})$, $4.73(\mathrm{~d}, J=10.4 \mathrm{~Hz}, 1 \mathrm{H}), 5.01(\mathrm{~d}, J=10.0 \mathrm{~Hz}, 1 \mathrm{H}), 6.83-7.38(\mathrm{~m}, 9 \mathrm{H}) .{ }^{13} \mathrm{C}-\mathrm{NMR}\left(\mathrm{CDCl}_{3}, 100 \mathrm{MHz}\right)$ : $\delta 24.1,52.8,56.4,77.7,116.8,120.6,127.4,127.7,128.5,128.9,129.0,129.1,138.3,154.3$. HRMS $\left(\mathrm{CI}^{+}\right)$: calculated for $\mathrm{C}_{16} \mathrm{H}_{18} \mathrm{NO}[\mathrm{M}+\mathrm{H}]^{+}, m / z 240.1389$; found for $[\mathrm{M}+\mathrm{H}]^{+}, m / z 240.1378$.

\subsubsection{3-Benzyl-4-butyl-3,4-dihydro-2H-1,3-benzoxazine (1c)}

According to the general procedure, a mixture of 2-\{1-(benzylamino)pentyl $\}$ phenol (1.0 g, $3.71 \mathrm{mmol})$ and formaldehyde $(0.14 \mathrm{~g}, 4.83 \mathrm{mmol}, 0.40 \mathrm{~mL})$ in dichloromethane $(15 \mathrm{~mL})$ was reacted. After purification $1 \mathrm{c}(1.01 \mathrm{~g}, 97 \%)$ was obtained as a colorless oil. ${ }^{1} \mathrm{H}-\mathrm{NMR}\left(\mathrm{CDCl}_{3}, 200 \mathrm{MHz}\right): \delta 0.86$ $(\mathrm{t}, J=7.0 \mathrm{~Hz}, 3 \mathrm{H}), 1.13-1.88(\mathrm{~m}, 6 \mathrm{H}), 3.48(\mathrm{dd}, J=9.8,3.8 \mathrm{~Hz}, 1 \mathrm{H}), 3.69(\mathrm{~d}, J=13.4 \mathrm{~Hz}, 1 \mathrm{H}), 4.00(\mathrm{~d}$, $J=13.4 \mathrm{~Hz}, 1 \mathrm{H}), 4.68(\mathrm{dd}, J=10.4,1.6 \mathrm{~Hz}, 1 \mathrm{H}), 4.96(\mathrm{~d}, J=10.4 \mathrm{~Hz}, 1 \mathrm{H}), 6.80-7.35(\mathrm{~m}, 9 \mathrm{H}) .{ }^{13} \mathrm{C}-\mathrm{NMR}$ $\left(\mathrm{CDCl}_{3}, 50 \mathrm{MHz}\right): \delta 14.2,22.5,28.5,37.9,56.9,57.2,77.8,116.6,120.4,124.9,127.4,127.6,128.4,128.8$, 129.3, 138.7, 153.7. HRMS $\left(\mathrm{CI}^{+}\right)$: calculated for $\mathrm{C}_{19} \mathrm{H}_{23} \mathrm{NO}[\mathrm{M}+\mathrm{H}]^{+}, m / z 282.1780$; found for $[\mathrm{M}+\mathrm{H}]^{+}$, $\mathrm{m} / \mathrm{z} 282.1788$.

\subsubsection{3-Benzyl-4-(s-butyl)-3,4-dihydro-2H-1,3-benzoxazine (1d)}

According to the general procedure, a mixture of 2-\{1-(benzylamino)-2-methylbutyl $\}$ phenol $(0.47 \mathrm{~g}$, $1.74 \mathrm{mmol})$ and formaldehyde $(0.06 \mathrm{~g}, 2.27 \mathrm{mmol}, 0.18 \mathrm{~mL})$ in dichloromethane $(10 \mathrm{~mL})$ was reacted. After purification $1 \mathrm{~d}(0.44 \mathrm{~g}, 91 \%)$ was obtained as a colorless oil. ${ }^{1} \mathrm{H}-\mathrm{NMR}\left(\mathrm{CDCl}_{3}, 400 \mathrm{MHz}\right): \delta 0.83(\mathrm{t}$, $J=7.2 \mathrm{~Hz}, 3 \mathrm{H}), 0.85\left(\mathrm{t}, J=7.6 \mathrm{~Hz}, 3 \mathrm{H}^{*}\right), 0.94(\mathrm{~d}, J=6.4 \mathrm{~Hz}, 3 \mathrm{H}), 0.98\left(\mathrm{~d}, J=6.4 \mathrm{~Hz}, 3 \mathrm{H}^{*}\right), 1.15-1.27(\mathrm{~m}, 3 \mathrm{H})$, $1.52-1.63\left(\mathrm{~m}, 1 \mathrm{H}^{*}\right), 1.69-1.85\left(\mathrm{~m}, 2 \mathrm{H}^{*}\right), 3.64(\mathrm{~d}, J=13.6 \mathrm{~Hz}, 1 \mathrm{H}), 3.66\left(\mathrm{~d}, J=13.2 \mathrm{~Hz}, 1 \mathrm{H}^{*}\right), 3.93(\mathrm{~d}, J=13.2$ $\mathrm{Hz}, 1 \mathrm{H}), 3.95\left(\mathrm{~d}, J=13.2 \mathrm{~Hz}, 1 \mathrm{H}^{*}\right), 4.68(\mathrm{dd}, J=10.4,0.8 \mathrm{~Hz}, 1 \mathrm{H}), 4.69\left(\mathrm{~d}, J=10.4 \mathrm{~Hz}, 1 \mathrm{H}^{*}\right), 4.97(\mathrm{~d}, J=10.0$ $\mathrm{Hz}, 1 \mathrm{H}), 5.00\left(\mathrm{~d}, J=10.0 \mathrm{~Hz}, 1 \mathrm{H}^{*}\right), 6.82-7.36\left(\mathrm{~m}, 9 \mathrm{H}, 9 \mathrm{H}^{*}\right) .{ }^{13} \mathrm{C}-\mathrm{NMR}\left(\mathrm{CDCl}_{3}, 100 \mathrm{MHz}\right): \delta 11.2,11.9^{*}$, $16.1,16.5^{*}, 25.5,26.1^{*}, 40.3,40.8^{*}, 57.5,57.8^{*}, 61.9,62.2^{*}, 78.0,78.5^{*}, 116.5,116.6^{*}, 116.9,119.3^{*}, 119.5,119.7^{*}$, $122.4,122.7^{*}, 127.4,127.9,128.0^{*}, 128.4^{*}, 129.4,129.5^{*}, 130.0,130.3^{*}, 138.7,138.8^{*}, 153.7,154.0^{*}$. HRMS (CI $)$ : calculated for $\mathrm{C}_{19} \mathrm{H}_{23} \mathrm{NO}[\mathrm{M}+\mathrm{H}]^{+}, m / z 282.1780$; found for $[\mathrm{M}+\mathrm{H}]^{+} m / z 282.1845$.

\subsubsection{3-Benzyl-4-phenyl-3,4-dihydro-2H-1,3-benzoxazine (1e)}

According to the general procedure, a mixture of 2-\{(benzylamino)(phenyl)methyl $\}$ phenol $(0.80 \mathrm{~g}$, $2.76 \mathrm{mmol})$ and formaldehyde $(0.10 \mathrm{~g}, 3.58 \mathrm{mmol}, 0.30 \mathrm{~mL})$ in dichloromethane $(15 \mathrm{~mL})$ was reacted. After crystallization in methanol $1 \mathrm{e}(1.04 \mathrm{~g}, 100 \%)$ was isolated as a white solid m.p. $=88-90{ }^{\circ} \mathrm{C}$. ${ }^{1} \mathrm{H}-\mathrm{NMR}\left(\mathrm{CDCl}_{3}, 400 \mathrm{MHz}\right): \delta 3.91(\mathrm{~d}, J=13.6 \mathrm{~Hz}, 1 \mathrm{H}), 4.06(\mathrm{~d}, J=13.6 \mathrm{~Hz}, 1 \mathrm{H}), 4.63(\mathrm{dd}, J=10.4$, $1.6 \mathrm{~Hz}, 1 \mathrm{H}), 4.76(\mathrm{~d}, J=9.6 \mathrm{~Hz}, 1 \mathrm{H}), 4.78(\mathrm{~s}, 1 \mathrm{H}), 6.88-7.44(\mathrm{~m}, 14 \mathrm{H}) .{ }^{13} \mathrm{C}-\mathrm{NMR}\left(\mathrm{CDCl}_{3}, 100 \mathrm{MHz}\right): \delta 56.7$, $60.2,78.1,116.9,120.4,128.3,128.5,128.6,129.2,129.4,138.6,143.6,154.3$. HRMS $\left(\mathrm{CI}^{+}\right)$: calculated for $\mathrm{C}_{21} \mathrm{H}_{19} \mathrm{NO}[\mathrm{M}+\mathrm{H}]^{+}, m / z$ 302.1576; found for $[\mathrm{M}+\mathrm{H}]^{+}, m / z 302.1561$. 


\subsubsection{3-Benzyl-4-(m-tolyl)-3,4-dihydro-2H-1,3-benzoxazine (1f)}

According to the general procedure, a mixture of 2-\{(benzylamino)( $m$-tolyl)methyl $\}$ phenol $(1.0 \mathrm{~g}$, $3.30 \mathrm{mmol})$ and formaldehyde $(0.12 \mathrm{~g}, 4.29 \mathrm{mmol}, 0.34 \mathrm{~mL})$ in dichloromethane $(15 \mathrm{~mL})$ was reacted. After purification $1 \mathrm{f}(0.89 \mathrm{~g}, 86 \%)$ was obtained as a colorless oil. ${ }^{1} \mathrm{H}-\mathrm{NMR}\left(\mathrm{CDCl}_{3}, 200 \mathrm{MHz}\right): \delta 2.31$ $(\mathrm{s}, 3 \mathrm{H}), 3.93(\mathrm{~d}, J=13.4 \mathrm{~Hz}, 1 \mathrm{H}), 4.08(\mathrm{~d}, J=13.2 \mathrm{~Hz}, 1 \mathrm{H}), 4.66(\mathrm{~d}, J=10.0 \mathrm{~Hz}, 1 \mathrm{H}), 4.77(\mathrm{~s}, 1 \mathrm{H}), 4.82(\mathrm{~d}$, $J=10.0 \mathrm{~Hz}, 1 \mathrm{H}), 6.93-7.49(\mathrm{~m}, 13 \mathrm{H}) .{ }^{13} \mathrm{C}-\mathrm{NMR}\left(\mathrm{CDCl}_{3}, 50 \mathrm{MHz}\right): \delta 21.7,56.7,60.4,78.1,116.8,120.4$, $126.3,127.7,128.4,128.5,128.6,129.5,129.9,130.0,130.2,130.3,137.9,138.5,143.5,154.3$. HRMS $\left(\mathrm{CI}^{+}\right)$: calculated for $\mathrm{C}_{22} \mathrm{H}_{21} \mathrm{NO}[\mathrm{M}+\mathrm{H}]^{+}, m / z$ 317.1780; found for $[\mathrm{M}+\mathrm{H}]^{+}, m / z 317.1830$.

\subsubsection{3-Benzyl-4-(p-tolyl)-3,4-dihydro-2H-1,3-benzoxazine (1g)}

According to the general procedure, a mixture of 2-\{(benzylamino)( $p$-tolyl)methyl $\}$ phenol $(0.3 \mathrm{~g}$, $0.98 \mathrm{mmol})$ and formaldehyde $(0.03 \mathrm{~g}, 1.27 \mathrm{mmol}, 0.10 \mathrm{~mL})$ in dichloromethane $(10 \mathrm{~mL})$ was reacted. After crystallization in methanol $1 \mathrm{~g}(0.23 \mathrm{~g}, 76 \%)$ was obtained as a white solid m.p. $=80-83^{\circ} \mathrm{C}$. ${ }^{1} \mathrm{H}-\mathrm{NMR}\left(\mathrm{CDCl}_{3}, 400 \mathrm{MHz}\right): \delta 2.31(\mathrm{~s}, 3 \mathrm{H}), 3.91(\mathrm{~d}, J=13.2 \mathrm{~Hz}, 1 \mathrm{H}), 4.06(\mathrm{~d}, J=13.6 \mathrm{~Hz}, 1 \mathrm{H}), 4.63(\mathrm{~d}, J$ $=10.0 \mathrm{~Hz}, 1 \mathrm{H}), 4.78(\mathrm{~s}, 1 \mathrm{H}), 4.82(\mathrm{~d}, J=10.0 \mathrm{~Hz}, 1 \mathrm{H}), 6.91-7.45(\mathrm{~m}, 13 \mathrm{H}) \cdot{ }^{13} \mathrm{C}-\mathrm{NMR}\left(\mathrm{CDCl}_{3}, 50 \mathrm{MHz}\right): \delta$ 21.1, 56.6, 60.4, 78.1, 116.9, 120.4, 126.3, 127.7, 128.5, 128.6, 129.2, 129.5, 129.9, 130.3, 137.2, 138.5, 143.5, 154.4. HRMS $\left(\mathrm{CI}^{+}\right)$: calculated for $\mathrm{C}_{22} \mathrm{H}_{21} \mathrm{NO}[\mathrm{M}+\mathrm{H}]^{+}, m / z 317.1780$; found for $[\mathrm{M}+\mathrm{H}]^{+}, m / z 317.1810$.

\subsubsection{3-Benzyl-4-(4-chlorophenyl)-3,4-dihydro-2H-1,3-benzoxazine (1h)}

According to the general procedure, a mixture of 2-\{(benzylamino)(4-chlorophenyl)-methyl $\}$ phenol $6(0.88 \mathrm{~g}, 2.73 \mathrm{mmol})$ and formaldehyde $(0.09 \mathrm{~g}, 3.27 \mathrm{mmol}, 0.26 \mathrm{~mL})$ in dichloromethane $(15 \mathrm{~mL})$ was reacted. After purification $1 \mathrm{~h}(0.58 \mathrm{~g}, 64 \%)$ was obtained as a colorless oil. ${ }^{1} \mathrm{H}-\mathrm{NMR}\left(\mathrm{CDCl}_{3}\right.$, $200 \mathrm{MHz}): \delta 3.88\left(\mathrm{~d},{ }^{2} J_{\mathrm{H}-\mathrm{H}}=13.2 \mathrm{~Hz}, 1 \mathrm{H}\right), 4.06\left(\mathrm{~d},{ }^{2} J_{\mathrm{H}-\mathrm{H}}=13.4 \mathrm{~Hz}, 1 \mathrm{H}\right), 4.68\left(\mathrm{~d},{ }^{2} J_{\mathrm{H}-\mathrm{H}}=11.2 \mathrm{~Hz}, 1 \mathrm{H}\right)$, $4.69\left(\mathrm{dd},{ }^{2} J_{\mathrm{H}-\mathrm{H}}=10.2,1.6 \mathrm{~Hz}, 1 \mathrm{H}\right), 5.29(\mathrm{~s}, 1 \mathrm{H}), 6.87-7.44(\mathrm{~m}, 13 \mathrm{H}) .{ }^{13} \mathrm{C}-\mathrm{NMR}\left(\mathrm{CDCl}_{3}, 50 \mathrm{MHz}\right): \delta 56.5$, $59.3,77.8,116.8,119.5,120.3,127.6,128.2,128.5,129.2,130.0,130.3,133.1,138.2,141.9,154.0$. HRMS $\left(\mathrm{CI}^{+}\right)$: calculated for $\mathrm{C}_{21} \mathrm{H}_{18} \mathrm{ClNO}[\mathrm{M}+\mathrm{H}]^{+}, m / z 335.1078$; found for $[\mathrm{M}+\mathrm{H}]^{+}, m / z 336.1156$.

3.3. General Procedure for Preparation of 2-hydroxybenzylphosphonates $2 \boldsymbol{b}, 2 \boldsymbol{e}, 2 \boldsymbol{g}$ and $\alpha$-Aminophosphonates $3 a, 3 d, 3 h$

A mixture of 1,3-benzoxazine 1a-h (1.0 eq.), triethyl phosphite (1.0 eq.) and boron trifluoride etherate $(20 \mathrm{~mol} \%)$ in acetonitrile was stirred under nitrogen atmosphere at $26^{\circ} \mathrm{C}$ for $72 \mathrm{~h}$. Then, the solvent was evaporated under reduced pressure. The crude was dissolved in dichloromethane $(1.0 \mathrm{~mL})$, a saturated solution of ammonium chloride $(1.0 \mathrm{~mL})$ was added and the reaction mixture was stirred for $15 \mathrm{~min}$. The organic phase was extracted with dichloromethane and dried with anhydrous sodium sulfate. Finally, the solvent was removed under reduced pressure and the crude was purified by flash chromatography using hexane:EtOAc (80:20).

\subsubsection{Diethyl-[1-(2-hydroxyphenyl)ethyl]phosphonate (2b)}

According to the general procedure, a mixture of 1,3-benzoxazine $1 \mathbf{b}(0.10 \mathrm{~g}, 0.41 \mathrm{mmol})$, triethyl phosphite $0.06 \mathrm{~g}(0.41 \mathrm{mmol}, 0.07 \mathrm{~mL})$ and boron trifluoride etherate $(0.01 \mathrm{~g}, 0.08 \mathrm{mmol}, 0.01 \mathrm{~mL})$ in acetonitrile $(3 \mathrm{~mL})$ was reacted. After purification $\mathbf{2 b}(0.03 \mathrm{~g}, 28 \%)$ was obtained as a colorless oil. ${ }^{1} \mathrm{H}-\mathrm{NMR}\left(\mathrm{CDCl}_{3}, 400 \mathrm{MHz}\right): \delta 1.21(\mathrm{t}, J=7.2 \mathrm{~Hz}, 3 \mathrm{H}), 1.23(\mathrm{t}, J=7.2 \mathrm{~Hz}, 3 \mathrm{H}), 1.57(\mathrm{dd}, J=18.0,7.6 \mathrm{~Hz}$, $3 \mathrm{H}), 3.85(\mathrm{dq}, J=23.6,7.6 \mathrm{~Hz}, 1 \mathrm{H}), 3.89-4.08(\mathrm{~m}, 4 \mathrm{H}), 6.87-7.20(\mathrm{~m}, 4 \mathrm{H}) .{ }^{13} \mathrm{C}-\mathrm{NMR}\left(\mathrm{CDCl}_{3}, 100 \mathrm{MHz}\right)$ : $\delta 13.2\left(\mathrm{~d},{ }^{2} J_{\mathrm{C}-\mathrm{P}}=4.4 \mathrm{~Hz}\right), 16.2,34.7\left(\mathrm{~d},{ }^{1} J_{\mathrm{C}-\mathrm{P}}=136.2 \mathrm{~Hz}\right), 63.0\left(\mathrm{~d},{ }^{2} J_{\mathrm{C}-\mathrm{P}}=7.3 \mathrm{~Hz}\right), 63.1\left(\mathrm{~d},{ }^{2} J_{\mathrm{C}-\mathrm{P}}=7.3 \mathrm{~Hz}\right)$, $119.5,120.9,124.3\left(\mathrm{~d},{ }^{2} J_{\mathrm{C}-\mathrm{P}}=7.4 \mathrm{~Hz}\right), 128.7\left(\mathrm{~d},{ }^{4} J_{\mathrm{C}-\mathrm{P}}=2.9 \mathrm{~Hz}\right), 129.2\left(\mathrm{~d},{ }^{5} J_{\mathrm{C}-\mathrm{P}}=7.3 \mathrm{~Hz}\right), 155.4\left(\mathrm{~d},{ }^{3} J_{\mathrm{C}-\mathrm{P}}=\right.$ $4.4 \mathrm{~Hz}) .{ }^{31} \mathrm{P}-\mathrm{NMR}\left(\mathrm{CDCl}_{3}, 161.90 \mathrm{MHz}\right): \delta 31.12$. HRMS $\left(\mathrm{CI}^{+}\right)$: calculated for $\mathrm{C}_{12} \mathrm{H}_{19} \mathrm{O}_{4} \mathrm{P}[\mathrm{M}+\mathrm{H}]^{+}$, $m / z$ 259.1099; found for $[\mathrm{M}+\mathrm{H}]^{+}, m / z 259.1110$. 


\subsubsection{Diethyl-[(2-hydroxyphenyl)(phenyl)methyl]phosphonate (2e)}

According to the general procedure, a mixture of 1,3-benzoxazine 1 e $(0.20 \mathrm{~g}, 0.66 \mathrm{mmol})$, triethylphosphite $(0.11 \mathrm{~g}, 0.66 \mathrm{mmol}, 0.11 \mathrm{~mL})$ and boron trifluoride etherate $(0.01 \mathrm{~g}, 0.13 \mathrm{mmol}$, $0.01 \mathrm{~mL})$ in acetonitrile $(5 \mathrm{~mL})$ was reacted. After purification $2 \mathbf{e}(0.08 \mathrm{~g}, 40 \%)$ was obtained as a white solid, m.p. $=157-159{ }^{\circ} \mathrm{C} .{ }^{1} \mathrm{H}-\mathrm{NMR}\left(\mathrm{CDCl}_{3}, 400 \mathrm{MHz}\right): \delta 1.12(\mathrm{t}, J=7.2 \mathrm{~Hz}, 3 \mathrm{H}), 1.15(\mathrm{t}, J=7.2 \mathrm{~Hz}$, $3 \mathrm{H}), 3.74-4.16(\mathrm{~m}, 4 \mathrm{H}), 4.72(\mathrm{~d}, J=26.6 \mathrm{~Hz}, 1 \mathrm{H}), 6.78-7.53(\mathrm{~m}, 9 \mathrm{H}) .{ }^{13} \mathrm{C}-\mathrm{NMR}\left(\mathrm{CDCl}_{3}, 100 \mathrm{MHz}\right): \delta 16.3$, $18.3,47.0\left(\mathrm{~d},{ }^{1} J_{\mathrm{C}-\mathrm{P}}=136.2 \mathrm{~Hz}\right), 63.5\left(\mathrm{~d},{ }^{2} J_{\mathrm{C}-\mathrm{P}}=7.0 \mathrm{~Hz}\right), 64.0\left(\mathrm{~d},{ }^{2} J_{\mathrm{C}-\mathrm{P}}=7.4 \mathrm{~Hz}\right), 119.5,121.0,123.7,129.1$, $129.5,129.7,130.0,131.1,131.2,132.5,137.2,155.3 .{ }^{31} \mathrm{P}-\mathrm{NMR}\left(\mathrm{CDCl}_{3}, 161.90 \mathrm{MHz}\right): \delta 28.78$. HRMS $\left(\mathrm{CI}^{+}\right)$: calculated for $\mathrm{C}_{17} \mathrm{H}_{21} \mathrm{O}_{4} \mathrm{P}[\mathrm{M}+\mathrm{H}]^{+}, \mathrm{m} / z$ 321.1256; found for $[\mathrm{M}+\mathrm{H}]^{+}, m / z 321.1306$.

\subsubsection{Diethyl-[(2-hydroxyphenyl)(p-tolyl)methyl]phosphonate (2g)}

According to the general procedure, a mixture of 1,3-benzoxazine $1 \mathrm{~g}(0.10 \mathrm{~g}, 0.31 \mathrm{mmol})$, triethyl phosphite $(0.05 \mathrm{~g}, 0.31 \mathrm{mmol}, 0.05 \mathrm{~mL})$ and boron trifluoride etherate $(0.009 \mathrm{~g}, 0.06 \mathrm{mmol}, 0.009 \mathrm{~mL})$ in acetonitrile $(3 \mathrm{~mL})$ was reacted. After purification $2 \mathrm{~g}(0.04 \mathrm{~g}, 30 \%)$ was obtained as a white solid, m.p. $=151-153^{\circ} \mathrm{C} .{ }^{1} \mathrm{H}-\mathrm{NMR}\left(\mathrm{CDCl}_{3}, 400 \mathrm{MHz}\right): \delta 1.13(\mathrm{t}, J=7.2 \mathrm{~Hz}, 3 \mathrm{H}), 1.16(\mathrm{t}, J=7.2 \mathrm{~Hz}, 3 \mathrm{H}), 2.32(\mathrm{~s}, 3 \mathrm{H})$, $3.88(\mathrm{~m}, 2 \mathrm{H}), 4.03(\mathrm{~m}, 2 \mathrm{H}), 4.69(\mathrm{~d}, J=26.4 \mathrm{~Hz}, 2 \mathrm{H}), 6.79-7.39(\mathrm{~m}, 8 \mathrm{H}) .{ }^{13} \mathrm{C}-\mathrm{NMR}\left(\mathrm{CDCl}_{3}, 100 \mathrm{MHz}\right)$ : $\delta 16.3,21.2,47.3\left(\mathrm{~d},{ }^{1} J_{\mathrm{C}-\mathrm{P}}=136.2 \mathrm{~Hz}\right), 63.4,64.0,119.5,121.0,123.7,129.1,129.5,129.7,130.0,131.1,131.2$, 132.5, 137.2, 155.3. ${ }^{31} \mathrm{P}-\mathrm{NMR}\left(\mathrm{CDCl}_{3}, 161.90 \mathrm{MHz}\right): \delta 28.78$. HRMS $\left(\mathrm{CI}^{+}\right)$: calculated for $\mathrm{C}_{18} \mathrm{H}_{23} \mathrm{O}_{4} \mathrm{P}[\mathrm{M}$ $+\mathrm{H}]^{+}, m / z 335.1412$; found for $[\mathrm{M}+\mathrm{H}]^{+}, m / z 335.1419$.

\subsubsection{Diethyl \{[benzyl(2-hydroxybenzyl)amino]methyl\}phosphonate (3a)}

According to the general procedure, a mixture of 1,3-benzoxazine 1a $(0.20 \mathrm{~g}, 0.88 \mathrm{mmol})$, triethylphosphite $(0.16 \mathrm{~g}, 0.88 \mathrm{mmol}, 0.15 \mathrm{~mL})$, and boron trifluoride etherate $(0.02 \mathrm{~g}, 0.17 \mathrm{mmol}$, $0.02 \mathrm{~mL})$, in acetonitrile $(5 \mathrm{~mL})$ was reacted. After purification $3 \mathrm{a}(0.28 \mathrm{~g}, 89 \%)$ was obtained as a colorless oil ${ }^{1} \mathrm{H}-\mathrm{NMR}\left(\mathrm{CDCl}_{3}, 200 \mathrm{MHz}\right): \delta 1.29(\mathrm{t}, J=7.0 \mathrm{~Hz}, 3 \mathrm{H}), 2.87(\mathrm{~d}, J=11.6 \mathrm{~Hz}, 2 \mathrm{H}), 3.75(\mathrm{~s}, 2 \mathrm{H})$, 3.95, (s, 2H), 4.05 (dq, $J=7.2,7.2 \mathrm{~Hz}, 4 \mathrm{H}), 6.72-7.33(\mathrm{~m}, 9 \mathrm{H}) .{ }^{13} \mathrm{C}-\mathrm{NMR}\left(\mathrm{CDCl}_{3}, 50 \mathrm{MHz}\right): \delta 16.5,16.6$, $47.9\left(\mathrm{~d},{ }^{1} J_{\mathrm{C}-\mathrm{P}}=158.3 \mathrm{~Hz}\right), 58.8(\mathrm{~d}, J=6.4 \mathrm{~Hz}), 59.1(\mathrm{~d}, J=9.9 \mathrm{~Hz}), 62.2,62.4,116.5,119.5,121.9,127.9$, 128.7, 129.3, 129.6, 129.9, 136.6, 157.4. ${ }^{31} \mathrm{P}-\mathrm{NMR}\left(\mathrm{CDCl}_{3}, 80.9 \mathrm{MHz}\right): \delta 21.92$. HRMS $\left(\mathrm{CI}^{+}\right)$: calculated for $\mathrm{C}_{19} \mathrm{H}_{26} \mathrm{NO}_{4} \mathrm{P}[\mathrm{M}+\mathrm{H}]^{+}, m / z 364.1679$; found for $[\mathrm{M}+\mathrm{H}]^{+}, m / z 364.1724$.

\subsubsection{Diethyl\{benzyl[1-(2-hydroxyphenyl)-2-methylbutyl]amino\}methyl)phosphonate (3d)}

According to the general procedure, a mixture of 1,3-benzoxazine 1d, $(0.20 \mathrm{~g}, 0.83 \mathrm{mmol})$, triethylphosphite $(0.15 \mathrm{~g}, 0.83 \mathrm{mmol}, 0.14 \mathrm{~mL})$ and boron trifluoride etherate $(0.02 \mathrm{~g}, 0.16 \mathrm{mmol}$, $0.02 \mathrm{~mL})$, in acetonitrile $(5 \mathrm{~mL})$ were reacted. After purification $3 \mathrm{~d}(0.12 \mathrm{~g}, 37 \%)$ was obtained as a colorless oil The compound was characterized as diastereomeric mixture. ${ }^{1} \mathrm{H}-\mathrm{NMR}\left(\mathrm{CDCl}_{3}, 400 \mathrm{MHz}\right)$ : $\delta 0.53(\mathrm{~d}, J=6.8 \mathrm{~Hz}, 3 \mathrm{H}), 0.72(\mathrm{dd}, J=5.2 \mathrm{~Hz}, 5.2 \mathrm{~Hz}, 3 \mathrm{H}), 0.93(\mathrm{t}, J=7.2 \mathrm{~Hz}, 3 \mathrm{H})^{*}, 1.09(\mathrm{~d}, J=6.8 \mathrm{~Hz}$, $3 \mathrm{H})^{*}, 1.22\left(\mathrm{t}, J=7.2 \mathrm{~Hz}, 3 \mathrm{H}, 3 \mathrm{H}^{*}\right), 1.23\left(\mathrm{t}, J=7.2 \mathrm{~Hz}, 3 \mathrm{H}, 3 \mathrm{H}^{*}\right), 1.91(\mathrm{dq}, J=7.2,2.8 \mathrm{~Hz}, 2 \mathrm{H}), 1.95(\mathrm{dq}, J=$ $7.2,2.8 \mathrm{~Hz}, 2 \mathrm{H})^{*}, 2.22-2.35\left(\mathrm{~m}, 1 \mathrm{H}, 1 \mathrm{H}^{*}\right), 3.26(\mathrm{dd}, J=16.8,3.2 \mathrm{~Hz}, 1 \mathrm{H}), 3.28(\mathrm{dd}, J=16.8,3.2 \mathrm{~Hz}, 1 \mathrm{H})^{*}$, $3.32\left(\mathrm{~d}, J=7.6 \mathrm{~Hz}, 1 \mathrm{H}, 1 \mathrm{H}^{*}\right), 3.59(\mathrm{~d}, J=16.0 \mathrm{~Hz}, 1 \mathrm{H}), 3.64\left(\mathrm{~d}, J=14 \mathrm{~Hz}, 2 \mathrm{H}, 2 \mathrm{H}^{*}\right), 3.68(\mathrm{dd}, J=14.0,4.0$ $\mathrm{Hz}, 1 \mathrm{H})^{*}, 4.04-4.14\left(\mathrm{~m}, 2 \mathrm{H}, 2 \mathrm{H}^{*}\right), 4.23-4.33\left(\mathrm{~m}, 2 \mathrm{H}, 2 \mathrm{H}^{*}\right), 6.89-7.33\left(\mathrm{~m}, 9 \mathrm{H}, 9 \mathrm{H}^{*}\right), 10.40\left(\mathrm{~s}, 1 \mathrm{H}, 1 \mathrm{H}^{*}\right)$. ${ }^{13} \mathrm{C}-\mathrm{NMR}\left(\mathrm{CDCl}_{3}, 100 \mathrm{MHz}\right): 10.7,11.2^{*}, 16.4,16.5,16.6,17.2^{*}, 25.9,27.3,33.5,33.9,44.9\left(\mathrm{~d},{ }^{1} \mathrm{~J}_{\mathrm{C}-\mathrm{P}}=121.8\right.$ $\mathrm{Hz}), 45.0\left(\mathrm{~d},{ }^{1} J_{\mathrm{C}-\mathrm{P}}=122.6 \mathrm{~Hz}\right)^{*}, 58.0,62.1,62.2^{*}, 71.1,71.8^{*}, 116.9,117.1^{*}, 119.0,119.1^{*}, 122.3,125.0^{*}$, $127.5,127.5^{*}, 128.5,128.5^{*}, 128.8,128.9^{*}, 129.2,129.2^{*}, 132.9,138.5^{*}, 148.0,148.1^{*} .{ }^{31} \mathrm{P}-\mathrm{NMR}\left(\mathrm{CDCl}_{3}\right.$, $161.90 \mathrm{MHz}): \delta 26.01,26.36^{*} \mathrm{HRMS}\left(\mathrm{CI}^{+}\right)$: calculated for $\mathrm{C}_{23} \mathrm{H}_{34} \mathrm{NO}_{4} \mathrm{P}[\mathrm{M}+\mathrm{H}]^{+}, m / z$ 420.2305; found for $[\mathrm{M}+\mathrm{H}]^{+}, m / z 420.2286$.

\subsubsection{Diethyl((benzyl((4-chlorophenyl)(2-hydroxyphenyl)methyl)amino)methyl)phosphonate (3h)}

According to the general procedure, a mixture of 1,3-benzoxazine $1 \mathrm{~h}(0.20 \mathrm{~g}, 0.59 \mathrm{mmol})$, triethylphosphite $(0.10 \mathrm{~g}, 0.59 \mathrm{mmol}, 0.1 \mathrm{~mL})$ and boron trifluoride etherate $(0.01 \mathrm{~g}, 0.11 \mathrm{mmol}, 0.01 \mathrm{~mL})$ 
in acetonitrile $(5 \mathrm{~mL})$ were reacted. After purification $3 \mathrm{~h}(0.01 \mathrm{~g}, 6 \%)$ was obtained as a colorless oil ${ }^{1} \mathrm{H}-$ $\operatorname{NMR}\left(\mathrm{CDCl}_{3}, 200 \mathrm{MHz}\right): \delta 1.29(\mathrm{t}, J=7.2 \mathrm{~Hz}, 6 \mathrm{H}), 3.22(\mathrm{dd}, J=16.6,7.0 \mathrm{~Hz}, 1 \mathrm{H}), 3.43(\mathrm{dd}, J=16.5$, $4.8 \mathrm{~Hz}, 1 \mathrm{H}), 3.73-4.37(\mathrm{~m}, 4 \mathrm{H}), 3.82(\mathrm{dd}, J=6.2,3.2 \mathrm{~Hz}, 2 \mathrm{H}), 4.10(\mathrm{dd}, J=7.9,7.2 \mathrm{~Hz}, 1 \mathrm{H}), 5.06(\mathrm{~s}, 1 \mathrm{H})$, 6.69-7.36 (m, 13H), $10.83(\mathrm{~s}, 1 \mathrm{H}) .{ }^{13} \mathrm{C}-\mathrm{NMR}\left(\mathrm{CDCl}_{3}, 50 \mathrm{MHz}\right): \delta 16.4,16.5,45.0\left(\mathrm{~d},{ }^{1} J_{\mathrm{C}-\mathrm{P}}=124.5 \mathrm{~Hz}\right)$, 57.6, $61.9\left(\mathrm{~d},{ }^{3} J_{\mathrm{C}-\mathrm{P}}=7.5 \mathrm{~Hz}\right), 67.0,122.4,122.5,125.6,127.6,128.5,128.7,129.5,130.12,132.5,133.3,137.0$, 138.0. ${ }^{31} \mathrm{P}-\mathrm{NMR}\left(\mathrm{CDCl}_{3}, 161.90 \mathrm{MHz}\right): \delta 24.64$. HRMS $\left(\mathrm{CI}^{+}\right)$: calculated for $\mathrm{C}_{25} \mathrm{H}_{29} \mathrm{ClNO}_{4} \mathrm{P}[\mathrm{M}+\mathrm{H}]^{+}$, $m / z$ 474.1603; found for $[\mathrm{M}+\mathrm{H}]^{+}, m / z 474.1653$.

\subsection{General Procedure for Preparation of $\alpha$-Aminophosphonates $3 \boldsymbol{a}-\boldsymbol{h}$}

A mixture of 1,3-benzoxazines 1a-h (1.0 eq.), diethyl phosphite (1.0 eq.) and boron trifluoride etherate (0.2 eq.) was stirred at $26^{\circ} \mathrm{C}$ for $48 \mathrm{~h}$ in acetonitrile, then, the solvent was evaporated under reduced pressure and re-dissolved in dichloromethane. Afterward, a saturated solution of ammonium chloride was added and the reaction mixture was stirred for $15 \mathrm{~min}$. Finally, the organic phase was extracted with dichloromethane and dried over anhydrous sodium sulfate. The solvent was eliminated under reduced pressure and the crude was purified by flash chromatography using hexane:EtOAc (80:20).

\subsubsection{Diethyl\{[benzyl(2-hydroxybenzyl)amino]methyl\}phosphonate (3a)}

According to the general procedure, a mixture of 1,3-benzoxazine $1 \mathrm{a}(0.3 \mathrm{~g}, 1.33 \mathrm{mmol})$, diethyl phosphite $(0.18 \mathrm{~g}, 1.33 \mathrm{mmol}, 0.17 \mathrm{~mL})$ and boron trifluoride etherate $(0.03 \mathrm{~g}, 0.26 \mathrm{mmol}, 0.03 \mathrm{~mL})$ in acetonitrile $(5 \mathrm{~mL})$ were reacted during $48 \mathrm{~h}$. The reaction crude was purified by flash chromatography using hexane:EtOAc (80:20). After purification $3 \mathbf{a}(0.46 \mathrm{~g}, 96 \%)$ was obtained as a colorless oil.

\subsubsection{Diethyl(\{benzyl[1-(2-hydroxyphenyl)ethyl]amino\}methyl)phosphonate (3b)}

According to the general procedure, a mixture of 1,3-benzoxazine $1 \mathbf{b}(0.10 \mathrm{~g}, 0.41 \mathrm{mmol})$, diethyl phosphite $(0.05 \mathrm{~g}, 0.41 \mathrm{mmol}, 0.07 \mathrm{~mL})$ and boron trifluoride etherate $(0.01 \mathrm{~g}, 0.08 \mathrm{mmol}, 0.01 \mathrm{~mL})$ in acetonitrile $(3 \mathrm{~mL})$ were reacted. After purification $3 \mathbf{b}(0.12 \mathrm{~g}, 80 \%)$ was obtained as a colorless oil. ${ }^{1} \mathrm{H}-\mathrm{NMR}\left(\mathrm{CDCl}_{3}, 200 \mathrm{MHz}\right): \delta 1.25(\mathrm{t}, J=7.0 \mathrm{~Hz}, 3 \mathrm{H}), 1.28(\mathrm{t}, J=7.0 \mathrm{~Hz}, 3 \mathrm{H}), 1.47(\mathrm{~d}, J=6.6 \mathrm{~Hz}, 1 \mathrm{H})$, $2.92(\mathrm{~d}, J=12.2 \mathrm{~Hz}, 2 \mathrm{H}), 3.48(\mathrm{~d}, J=12.8 \mathrm{~Hz}, 1 \mathrm{H}), 3.79-4.19(\mathrm{~m}, 4 \mathrm{H}), 4.45(\mathrm{q}, J=7.0 \mathrm{~Hz}, 1 \mathrm{H}), 6.78-7.34$ $(\mathrm{m}, 9 \mathrm{H}) .{ }^{13} \mathrm{C}-\mathrm{NMR}\left(\mathrm{CDCl}_{3}, 50 \mathrm{MHz}\right): \delta 10.3,16.4,16.6,44.2\left(\mathrm{~d},{ }^{1} J_{\mathrm{C}-\mathrm{P}}=159.1 \mathrm{~Hz}\right), 54.9\left(\mathrm{~d},{ }^{3} J_{\mathrm{C}-\mathrm{P}}=7.6 \mathrm{~Hz}\right)$, $57.0\left(\mathrm{~d},{ }^{3} J_{\mathrm{C}-\mathrm{P}}=6.0 \mathrm{~Hz}\right), 62.2\left(\mathrm{~d},{ }^{2} J_{\mathrm{C}-\mathrm{P}}=9.1 \mathrm{~Hz}\right), 62.4\left(\mathrm{~d},{ }^{2} J_{\mathrm{C}-\mathrm{P}}=7.5 \mathrm{~Hz}\right), 116.7,119.3,126.5,127.3,127.8$, 128.6, 129.0, 129.8, 137.0, 157.1. ${ }^{31} \mathrm{P}-\mathrm{NMR}\left(\mathrm{CDCl}_{3}, 80.9 \mathrm{MHz}\right): \delta 26.19$. HRMS $\left(\mathrm{CI}^{+}\right)$: calculated for $\mathrm{C}_{20} \mathrm{H}_{28} \mathrm{NO}_{4} \mathrm{P}[\mathrm{M}+\mathrm{H}]^{+}, m / z 378.1756$; found for $[\mathrm{M}+\mathrm{H}]^{+}, m / z 378.1783$.

\subsubsection{Diethyl(\{benzyl[1-(2-hydroxyphenyl)pentyl]amino\}methyl)phosphonate (3c)}

According to the general procedure, a mixture of 1,3-benzoxazine $1 \mathrm{c}(0.30 \mathrm{~g}, 1.06 \mathrm{mmol})$, diethyl phosphite $(0.14 \mathrm{~g}, 1.06 \mathrm{mmol}, 0.13 \mathrm{~mL})$ and boron trifluoride etherate $(0.03 \mathrm{~g}, 0.21 \mathrm{mmol}, 0.03$ $\mathrm{mL})$ in acetonitrile $(5 \mathrm{~mL})$ were reacted. After purification $3 \mathrm{c}(0.26 \mathrm{~g}, 58 \%)$ was obtained as a colorless oil. ${ }^{1} \mathrm{H}-\mathrm{NMR}\left(\mathrm{CDCl}_{3}, 200 \mathrm{MHz}\right): \delta 0.90(\mathrm{t}, J=7.2 \mathrm{~Hz}, 3 \mathrm{H}), 1.27(\mathrm{t}, J=6.8 \mathrm{~Hz}, 3 \mathrm{H}), 1.28(\mathrm{t}, J=7.2 \mathrm{~Hz}$, $3 \mathrm{H}), 1.37(\mathrm{q}, J=7.2 \mathrm{~Hz}, 2 \mathrm{H}), 1.78-1.88(\mathrm{~m}, 2 \mathrm{H}), 1.95-2.05(\mathrm{~m}, 2 \mathrm{H}), 2.91(\mathrm{~d}, J=16.0 \mathrm{~Hz}, 1 \mathrm{H}), 3.00(\mathrm{dd}, J=$ 16.0, $7.2 \mathrm{~Hz}, 1 \mathrm{H}), 3.64(\mathrm{~d}, J=13.2 \mathrm{~Hz}, 1 \mathrm{H}), 3.92-4.07(\mathrm{~m}, 4 \mathrm{H}), 4.03(\mathrm{~d}, J=13.2 \mathrm{~Hz}, 1 \mathrm{H}), 4.15(\mathrm{~d}, J=10.0$ $\mathrm{Hz}, 2 \mathrm{H}), 6.81-7.34(\mathrm{~m}, 9 \mathrm{H}), 9.60(\mathrm{~s}, 1 \mathrm{H}) .{ }^{13} \mathrm{C}-\mathrm{NMR}\left(\mathrm{CDCl}_{3}, 100 \mathrm{MHz}\right): \delta 14.2,16.5,16.6,23.1,26.0,29.5$, $44.4\left(\mathrm{~d},{ }^{1} J_{\mathrm{C}-\mathrm{P}}=155.3 \mathrm{~Hz}\right), 55.1\left(\mathrm{~d},{ }^{3} J_{\mathrm{C}-\mathrm{P}}=7.3 \mathrm{~Hz}\right), 62.1\left(\mathrm{~d},{ }^{2} J_{\mathrm{C}-\mathrm{P}}=5.8 \mathrm{~Hz}\right), 62.3\left(\mathrm{~d},{ }^{2} J_{\mathrm{C}-\mathrm{P}}=5.9 \mathrm{~Hz}\right), 63.2$, 117.2, 119.2, 125.2, 127.8, 128.5, 128.7, 128.9, 129.9, 137.6, 157.3. ${ }^{31}$ P-NMR $\left(\mathrm{CDCl}_{3}, 161.90 \mathrm{MHz}\right): \delta 25.71$. HRMS $\left(\mathrm{CI}^{+}\right)$: calculated for $\mathrm{C}_{23} \mathrm{H}_{34} \mathrm{NO}_{4} \mathrm{P}[\mathrm{M}+\mathrm{H}]^{+}, m / z$ 420.2305; found for $[\mathrm{M}+\mathrm{H}]^{+}, m / z 420.2355$.

\subsubsection{Diethyl\{benzyl[1-(2-hydroxyphenyl)-2-methylbutyl]amino\}methyl)phosphonate (3d)}

According to the general procedure, a mixture of 1,3-benzoxazine $1 \mathrm{~d}(0.30 \mathrm{~g}, 1.06 \mathrm{mmol})$, diethyl phosphite $(0.14 \mathrm{~g}, 1.06 \mathrm{mmol}, 0.13 \mathrm{~mL})$ and boron trifluoride etherate $(0.03 \mathrm{~g}, 0.21 \mathrm{mmol}, 0.03$ 
$\mathrm{mL})$ in acetonitrile $(5 \mathrm{~mL})$ was reacted. After purification $3 \mathrm{~d}(0.16 \mathrm{~g}, 37 \%)$ was obtained as a colorless oil. The compound was characterized as diastereomeric mixture.

\subsubsection{Diethyl(\{benzyl[(2-hydroxyphenyl)(phenyl)methyl]amino\}methyl)phosphonate (3e)}

According to the general procedure, a mixture of 1,3-benzoxazine $1 \mathbf{e}(0.20 \mathrm{~g}, 0.66 \mathrm{mmol})$, diethyl phosphite $(0.09 \mathrm{~g}, 0.66 \mathrm{mmol}, 0.08 \mathrm{~mL})$ and boron trifluoride etherate $(0.018 \mathrm{~g}, 0.13 \mathrm{mmol}, 0.016 \mathrm{~mL})$ in acetonitrile $(5 \mathrm{~mL})$ was reacted. After purification $3 \mathbf{e}(0.19 \mathrm{~g}, 65 \%)$ was obtained as s colorless oil. ${ }^{1} \mathrm{H}-\mathrm{NMR}\left(\mathrm{CDCl}_{3}, 400 \mathrm{MHz}\right): \delta 1.25(\mathrm{t}, J=7.2 \mathrm{~Hz}, 3 \mathrm{H}), 1.31(\mathrm{t}, J=7.2 \mathrm{~Hz}, 3 \mathrm{H}), 2.86(\mathrm{dd}, J=15.6,5.6$ $\mathrm{Hz}, 1 \mathrm{H}), 2.99(\mathrm{dd}, J=18.0,15.6 \mathrm{~Hz}, 1 \mathrm{H}), 3.64(\mathrm{~d}, J=13.2 \mathrm{~Hz}, 1 \mathrm{H}), 4.14(\mathrm{~d}, J=12.8 \mathrm{~Hz}, 1 \mathrm{H}), 3.85-4.00$ $(\mathrm{m}, 2 \mathrm{H}), 4.04-4.14(\mathrm{~m}, 2 \mathrm{H}), 5.48(\mathrm{~s}, 1 \mathrm{H}), 6.70-7.47(\mathrm{~m}, 14 \mathrm{H}), 10.74(\mathrm{~s}, 1 \mathrm{H}) .{ }^{13} \mathrm{C}-\mathrm{NMR}\left(\mathrm{CDCl}_{3}, 100 \mathrm{MHz}\right)$ : $\delta 16.4,16.6,44.4\left(\mathrm{~d},{ }^{1} J_{\mathrm{C}-\mathrm{P}}=156.0 \mathrm{~Hz}\right), 55.1,\left(\mathrm{~d},{ }^{2} J_{\mathrm{C}-\mathrm{P}}=6.5 \mathrm{~Hz}\right), 62.2\left(\mathrm{~d},{ }^{2} J_{\mathrm{C}-\mathrm{P}}=5.5 \mathrm{~Hz}\right), 69.3\left(\mathrm{~d},{ }^{2} J_{\mathrm{C}-\mathrm{P}}=6.5\right.$ Hz), 117.1, 119.4, 124.6, 124.8, 127.9, 128.5, 128.7, 128.9, 129.1, 129.9, 130.2, 130.4, 136.4, 157.2. ${ }^{31} \mathrm{P}-\mathrm{NMR}$ $\left(\mathrm{CDCl}_{3}, 161.90 \mathrm{MHz}\right): \delta 27.89$. HRMS $\left(\mathrm{CI}^{+}\right)$: calculated for $\mathrm{C}_{25} \mathrm{H}_{30} \mathrm{NO}_{4} \mathrm{P}[\mathrm{M}+\mathrm{H}]^{+}, m / z$ 440.1992; found for $[\mathrm{M}+\mathrm{H}]^{+}, m / z 440.2004$.

\subsubsection{Diethyl(\{benzyl[(2-hydroxyphenyl)(m-tolyl)methyl]amino\}methyl)phosphonate (3f)}

According to the general procedure, a mixture of 1,3-benzoxazine $1 \mathrm{f}(0.3 \mathrm{~g}, 0.95 \mathrm{mmol})$, diethyl phosphite $(0.13 \mathrm{~g}, 0.95 \mathrm{mmol}, 0.12 \mathrm{~mL})$ and boron trifluoride etherate $(0.027 \mathrm{~g}, 0.19 \mathrm{mmol}, 0.024 \mathrm{~mL})$ in acetonitrile $(6 \mathrm{~mL})$ was reacted. After purification $3 \mathbf{f}(0.15 \mathrm{~g}, 35 \%)$ was obtained as a colorless oil. ${ }^{1} \mathrm{H}-$ NMR (CDCl $3,400 \mathrm{MHz}): \delta 1.25(\mathrm{t}, J=7.2 \mathrm{~Hz}, 3 \mathrm{H}), 1.32(\mathrm{t}, J=7.2 \mathrm{~Hz}, 3 \mathrm{H}), 2.37(\mathrm{~s}, 3 \mathrm{H}), 2.87(\mathrm{dd}, J=15.6$, $5.6 \mathrm{~Hz}, 1 \mathrm{H}), 2.99(\mathrm{dd}, J=18.0,15.6 \mathrm{~Hz}, 1 \mathrm{H}), 3.66(\mathrm{~d}, J=13.2 \mathrm{~Hz}, 1 \mathrm{H}), 3.82-4.02(\mathrm{~m}, 2 \mathrm{H}), 4.04-4.17(\mathrm{~m}$, 2H), $4.09(\mathrm{~d}, J=13.2 \mathrm{~Hz}, 1 \mathrm{H}), 5.41(\mathrm{~s}, 1 \mathrm{H}), 6.70-7.47(\mathrm{~m}, 13 \mathrm{H}), 10.79(\mathrm{~s}, 1 \mathrm{H}) .{ }^{13} \mathrm{C}-\mathrm{NMR}(\mathrm{CDCl}, 100 \mathrm{MHz})$ : $\delta 16.5\left(\mathrm{~d},{ }^{3} J_{\mathrm{C}-\mathrm{P}}=5.0 \mathrm{~Hz}\right), 16.6\left(\mathrm{~d},{ }^{3} J_{\mathrm{C}-\mathrm{P}}=5.0 \mathrm{~Hz}\right), 44.4\left(\mathrm{~d},{ }^{1} J_{\mathrm{C}-\mathrm{P}}=155.0 \mathrm{~Hz}\right), 55.1,\left(\mathrm{~d},{ }^{3} J_{\mathrm{C}-\mathrm{P}}=6.0 \mathrm{~Hz}\right), 62.3$ $\left(\mathrm{d},{ }^{2} J_{\mathrm{C}-\mathrm{P}}=5.0 \mathrm{~Hz}\right), 69.5\left(\mathrm{~d},{ }^{2} J_{\mathrm{C}-\mathrm{P}}=6.0 \mathrm{~Hz}\right), 117.1,119.4,124.7,127.3,127.9,128.6,128.7,128.9,129.1$, 129.2, 130.0, 130.3, 131.0, 136.5, 138.5, 157.2. ${ }^{31} \mathrm{P}-\mathrm{NMR}\left(\mathrm{CDCl}_{3}, 161.90 \mathrm{MHz}\right): \delta 28.52 . \mathrm{HRMS}\left(\mathrm{CI}^{+}\right)$: calculated for $\mathrm{C}_{26} \mathrm{H}_{32} \mathrm{NO}_{4} \mathrm{P}[\mathrm{M}+\mathrm{H}]^{+}, m / z$ 454.5404; found for $[\mathrm{M}+\mathrm{H}]^{+}, m / z 454.5454$.

\subsubsection{Diethyl(\{benzyl[(2-hydroxyphenyl)(p-tolyl)methyl]amino\}methyl)phosphonate (3g)}

According to the general procedure, a mixture of 1,3-benzoxazine $1 \mathrm{~g}(0.25 \mathrm{~g}, 0.79 \mathrm{mmol})$, diethyl phosphite $(0.10 \mathrm{~g}, 0.79 \mathrm{mmol}, 0.10 \mathrm{~mL})$ and boron trifluoride etherate $(0.02 \mathrm{~g}, 0.15 \mathrm{mmol}, 0.02 \mathrm{~mL})$ in acetonitrile $(5 \mathrm{~mL})$ was reacted. After purification $3 \mathbf{g}(0.086 \mathrm{~g}, 24 \%)$ was obtained as a colorless oil. ${ }^{1} \mathrm{H}-\mathrm{NMR}\left(\mathrm{CDCl}_{3}, 400 \mathrm{MHz}\right): \delta 1.25(\mathrm{t}, J=7.2 \mathrm{~Hz}, 3 \mathrm{H}), 1.32(\mathrm{t}, J=7.2 \mathrm{~Hz}, 3 \mathrm{H}), 2.37(\mathrm{~s}, 3 \mathrm{H}), 2.84(\mathrm{dd}, J$ $=15.6,5.2 \mathrm{~Hz}, 1 \mathrm{H}), 2.98(\mathrm{dd}, J=18,16 \mathrm{~Hz}, 1 \mathrm{H}), 3.60(\mathrm{~d}, J=13.2 \mathrm{~Hz}, 2 \mathrm{H}), 3.82-4.00(\mathrm{~m}, 2 \mathrm{H}), 4.044 .15$ $(\mathrm{m}, 2 \mathrm{H}), 5.44(\mathrm{~s}, 1 \mathrm{H}), 6.69-7.36(\mathrm{~m}, 13 \mathrm{H}), 10.83(\mathrm{~s}, 1 \mathrm{H}) .{ }^{13} \mathrm{C}-\mathrm{NMR}\left(\mathrm{CDCl}_{3}, 100 \mathrm{MHz}\right): \delta 16.5,16.6,44.4$ $\left(\mathrm{d},{ }^{1} J_{\mathrm{C}-\mathrm{P}}=157.0 \mathrm{~Hz}\right), 55.1,\left(\mathrm{~d},{ }^{3} J_{\mathrm{C}-\mathrm{P}}=7.0 \mathrm{~Hz}\right), 62.3\left(\mathrm{~d},{ }^{2} J_{\mathrm{C}-\mathrm{P}}=7.0 \mathrm{~Hz}\right), 69.0\left(\mathrm{~d},{ }^{2} J_{\mathrm{C}-\mathrm{P}}=7.0 \mathrm{~Hz}\right), 117.1$, 119.3, 124.7, 127.3, 127.9, 128.7, 129.1, 129.5, 130.0, 130.3, 130.4, 136.5, 138.2, 157.3. ${ }^{31} \mathrm{P}-\mathrm{NMR}\left(\mathrm{CDCl}_{3}\right.$, $161.90 \mathrm{MHz}): \delta$ 22.55. HRMS $\left(\mathrm{CI}^{+}\right)$: calculated for $\mathrm{C}_{26} \mathrm{H}_{32} \mathrm{NO}_{4} \mathrm{P}[\mathrm{M}+\mathrm{H}]^{+}, m / z$ 454.5404; found for $[\mathrm{M}+\mathrm{H}]^{+}, m / z 454.5444$.

\subsubsection{Diethyl ((benzyl((4-chlorophenyl)(2-hydroxyphenyl)methyl)amino)methyl)phosphonate (3h)}

According to the general procedure, a mixture of 1,3-benzoxazine $1 \mathrm{~h}(0.21 \mathrm{~g}, 0.63 \mathrm{mmol})$, diethyl phosphite $(0.086 \mathrm{~g}, 0.63 \mathrm{mmol}, 0.081 \mathrm{~mL})$ and boron trifluoride etherate $(0.017 \mathrm{~g}, 0.12 \mathrm{mmol}$, $0.02 \mathrm{~mL})$ in acetonitrile $(5 \mathrm{~mL})$ was reacted. After purification $3 \mathbf{h}(0.15 \mathrm{~g}, 50 \%)$ was obtained as a colorless oil.

\subsection{2-[(3-Cchloropropoxy)(phenyl)methyl]phenol (5e)}

A mixture of 1,3-benzoxazine 1 e $(0.15 \mathrm{~g}, 5 \mathrm{mmol})$ and 3-chloro-1-propanol $(0.5 \mathrm{~mL}, 0.56 \mathrm{~g}, 5.9 \mathrm{mmol})$ was stirred at $70{ }^{\circ} \mathrm{C}$ for $12 \mathrm{~h}$ at $70{ }^{\circ} \mathrm{C}$. Then, the crude was purified by flash chromatography using hexane:EtOAc (80:20), to give 5e $(0.072 \mathrm{~g}, 53 \%){ }^{1} \mathrm{H}-\mathrm{NMR}\left(\mathrm{CDCl}_{3}, 200 \mathrm{MHz}\right): \delta 2 .(\mathrm{dq}, J=6.2,1.6 \mathrm{~Hz}$, 
2H), $3.80(\mathrm{~m}, 4 \mathrm{H}), 5.55$ (s, 1H), $7.02(\mathrm{~m}, 9 \mathrm{H}), 7.80$ (br, $1 \mathrm{H}) .{ }^{13} \mathrm{C}-\mathrm{NMR}\left(\mathrm{CDCl}_{3}, 50 \mathrm{MHz}\right): \delta 32.6,41.7$, 66.4, 85.2, 117.3, 120.0, 124.9, 127.3, 127.6, 128.4, 128.8, 129.0, 129.4, 129.6, 139.9, 155.5. HRMS (CI $\left.{ }^{+}\right)$: calculated for $\mathrm{C}_{16} \mathrm{H}_{17} \mathrm{O}_{2} \mathrm{Cl}[\mathrm{M}+\mathrm{H}]^{+}, m / z: 277.0917$; found for $[\mathrm{M}+\mathrm{H}]^{+}, m / z 277.0880$.

\section{Conclusions}

We have developed a novel "one-pot" method for the synthesis of secondary benzyl phosphonates and $\alpha$-aminophosphonates from 1,3-benzoxazines. The phosphonates were obtained through direct $o$-QM formation, followed by a phospha-Michael addition reaction and the $\alpha$-aminophosphonates by iminium ion formation and the subsequent alkylphophites addition. In addition, this synthetic methodology was used to the preparation a valuable $o$-hydroxybenzyl ether derivative, which makes it a useful and efficient method for the synthesis of phosphonates, $\alpha$-aminophosphonates and benzyl ethers.

Author Contributions: I.L.-E. provided the concepts of the work, interpreted the results and prepared the manuscript. O.S.-E., A.H.-G., I.R.-E., they carried out the experimental work and interpreted the results. All authors read and approved the final manuscript.

Acknowledgments: The authors thank the Consejo Nacional de Ciencia y Tecnología (CONACYT) of México for financial support through project 807 and Laboratorio Nacional de Estructura de Macromoléculas (LANEM) as well O.S.-E. thank CONACYT for Graduate Scholarship 248543. I.R.-E. also thank CONACYT for Cátedra contract 942. We thank Blanca E. Domínguez-Mendoza and V. Labastida-Galván for the determination of the NMR spectra and HRMS.

Conflicts of Interest: The authors declare no conflict of interest.

\section{References}

1. Moonen, K.; Laureyn, I.; Stevens, C.V. Synthetic methods azaheterocyclic phosphonates and their biological activity. Chem. Rev. 2004, 104, 6177-6215. [CrossRef] [PubMed]

2. Wu, D.; Niu, J.Q.; Ding, Y.H.; Wu, X.Y.; Zhong, B.H.; Feng, X.W. Antiviral effects of three novel derivatives of adefovir on the replication of hepatitis B virus. Med. Chem. Res. 2012, 21, 1179-1187. [CrossRef]

3. Kafarski, P.; Lejczak, B. Biological activity of aminophosphonic acids. Phosphorus Sulfur Silicon Relat. Element. 1991, 63, 193-215. [CrossRef]

4. Sasaki, M. Current status of organophosphorus insecticide and stereochemistry. Phosphorus Sulfur Silicon Relat. Element. 2008, 183, 291-299. [CrossRef]

5. Lindsay, C.I.; Hill, S.B.; Hearn, M.; Manton, M.; Everall, N.; Bunn, A.; Heron, J.; Fletcher, I. Mechanism of action of phosphorus based flame retardants in acrylic polymers. Polym. Int. 2000, 49, 1183-1192. [CrossRef]

6. Kafarski, P.; Lejczak, B. Aminophosphonic acids of potential medical importance. Curr. Med. Chem. 2001, 1, 301-312. [CrossRef]

7. Lejczak, B.; Kafarski, P. Biological activity of aminophosphonic acids and their short peptides. Top. Heterocycl. Chem. 2009, 20,3-63. [CrossRef]

8. Naydenova, E.D.; Todorov, P.T.; Troev, K.D. Recent synthesis of aminophosphonic acids as potential biological importance. Amino Acids 2010, 38, 23-30. [CrossRef]

9. Orsini, F.; Sello, G.; Sisti, M. Aminophosphonic acids and derivatives. Synthesis and biological applications. Curr. Med. Chem. 2010, 17, 264-289. [CrossRef]

10. Moszner, N.; Zeuner, F.; Fisher, U.K.; Rheinberger, V. Monomers for adhesive polymers, 2. Synthesis and radical polymerisation of hydrolytically stable acrylic phosphonic acids. Macromol. Chem. Phys. 1999, 200, 1062-1067. [CrossRef]

11. Cabasso, I.; Smid, J.; Sahni, S.K. Radiopaque polymers based on acrylated phosphonate esters derived from polyols. J. Appl. Polym. Sci. 1990, 41, 3025-3042. [CrossRef]

12. Salasi, M.; Sharabi, T.; Roayaei, E.; Aliofkhazraei, M. The electrochemical behavior of environment-friendly inhibitors of silicate and phosphonate in corrosion control of carbon steel in soft water media. Mater. Chem. Phys. 2007, 104, 183-190. [CrossRef]

13. Kavipriya, K.; Rajendran, S.; Sathiyabama, J.; Prabha, A.S. A critical review of corrosion inhibition by phosphonic acids. Eur. Chem. Bull. 2012, 1, 366-374. [CrossRef] 
14. Knepper, T.P. Synthetic chelating agents and compounds exhibiting complexing properties in aquatic environment. Trends Analytic. Chem. 2003, 22, 708-724. [CrossRef]

15. Canadell, J.; Hunt, B.J.; CooK, A.G.; Mantecon, A.; Cadiz, V. Flame retardance and shrinkage reduction of polystyrene modified with acrylate-containing phosphorus and crosslinkable spiro-orthoester moieties. Polym. Degrad. Stab. 2007, 92, 1482-1490. [CrossRef]

16. Singh, H.; Jain, A.K. Ignition, combustion, toxicity, and fire retardancy of polyurethane foams: A comprehensive review. J. Appl. Polym. Sci. 2009, 111, 1115-1143. [CrossRef]

17. Ai, H.; Xu, K.; Liu, H.; Chen, M.; Zhang, X. Synthesis, characterization, and curing properties of novel phosphorus-containing naphthyl epoxy systems. J. Appl. Polym. Sci. 2009, 113, 541-546. [CrossRef]

18. Herrera-Taboada, L.; Guzmann, M.; Neubecker, K.; Goethlich, A. Process and Polymer for Preventing Ba/Sr Scale with a Detectable Phosphorus Funtionality. U.S. Patent US 12/520,642, 8 July 2010.

19. Demmer, Ch.S.; Krogsgaard-Larsen, N.; Bunch, L. Review on modern advances of chemical methods for the introduction of a phosphonic acid group. Chem. Rev. 2011, 111, 7981-8006. [CrossRef]

20. Ordoñez, M.; Rojas-Cabrera, H.; Cativiela, C. An overview of stereoselective synthesis of $\alpha$-aminophosphonic acids and derivates. Tetrahedron 2009, 65, 17-49. [CrossRef]

21. Ma, X.; Xu, Q.; Li, H.; Su, Ch.; Yu, L.; Zhang, X.; Cao, H.; Han, L.B. Alcohol-based Michaelis-Arbuzov reaction: An efficient and environmentally-benign method for $\mathrm{C}-\mathrm{P}(\mathrm{O})$ bond formation. Green Chem. 2018, 20, 3408-3413. [CrossRef]

22. Sharova, E.V.; Artyushin, O.I.; Odinets, I.L. Synthetic routes to carbamoylmethylphosphoryl compounds-extractants for the processing of spent nuclear fuels. Russ. Chem. Rev. 2014, 83, 95-119. [CrossRef]

23. Fan, W.; Queneau, Y.; Popowycz, F. The synthesis of HMF-based $\alpha$-amino phosphonates via one-pot Kabachnik-Fields reaction. RSC Adv. 2018, 8, 31496-31501. [CrossRef]

24. Fields, E.K. The Synthesis of esters of substituted amino phosphonic Acids. J. Am. Chem. Soc. 1952, 74, 1528-1531. [CrossRef]

25. Cytlak, T.; Skibinska, M.; Kaczmarek, P.; Kazmierczak, M.; Rapp, M.; Kubickia, M.; Koroniak, H. Functionalization of $\alpha$-hydroxyphosphonates as a convenient route to $N$-tosyl- $\alpha$-aminophosphonates. RSC Adv. 2018, 8, 11957-11974. [CrossRef]

26. Bálint, E.; Tajti, A.; Anna Ádám, A.; Csontos, I.; Karaghioso, K.; Czugle, M.; Ábrányi-Balogh, P.; Keglevich, $\mathrm{G}$. The synthesis of $\alpha$-aryl- $\alpha$-aminophosphonates and $\alpha$-aryl- $\alpha$-aminophosphine oxides by the microwave-assisted Pudovik reaction. Beilstein J. Org. Chem. 2017, 13, 76-86. [CrossRef] [PubMed]

27. Ordóñez, M.; Viveros-Ceballos, J.L.; Romero-Estudillo, I. Stereoselective Synthesis of $\alpha$-Aminophosphonic Acids through Pudovik and Kabachnik-Fields Reaction. In Amino Acid-New Insights and Roles in Plant and Animal, 1st ed.; Asao, T., Asaduzzaman, M., Eds.; InTechOpen: Rijeka, Croatia, 2017; Chapter 6; pp. 127-151. ISBN 978-953-51-3242-4.

28. Chen, Z.; Shi, Q.; Wang, G.; Chen, S.; Hu, J. Straightforward synthesis of bifunctional phosphorus phenols via phosphination of in situ generated $o$-quinine methides. Molecules 2018, 23, 1240. [CrossRef] [PubMed]

29. Huang, H.; Kang, J.Y. Organocatalytic phosphonylation of in situ formed o-quinone methides. Org. Lett. 2017, 19, 5988-5991. [CrossRef]

30. Perez-Prieto, J.; Galian, R.E.; Miranda, M.A.; Catalina, F.; Martín-Vargas, N.; López-Ortiz, F. Benzo[d]1,2-oxaphospholes as precursors of stabilized C-centered radicals. Org. Lett. 2004, 6, 561-564. [CrossRef]

31. Pérez-Prieto, J.; Galian, R.E.; Oña-Burgos, P.; Morant-Miñana, M.C.; Miranda, M.A.; López-Ortiz, F. Influence of substitution at the benzylic position on the behavior of stereoisomeric phosphorus compounds as precursors of stabilized carbon-centered radicals. Org. Lett. 2005, 7, 3869-3872. [CrossRef]

32. Kalla, R.M.N.; Lee, H.R.; Cao, J.; Yoo, J.W.; Kim, I. Phospho sulfonic acid: An efficient and reciclable solid acid catalyst for the solvent-free synthesis of $\alpha$-hydroxyphosphonates and their anticancer properties. New J. Chem. 2015, 39, 3916-3922. [CrossRef]

33. Salgado-Escobar, O.; Chavelas-Hernández, L.; Domínguez-Mendoza, B.E.; Linzaga-Elizalde, I.; Ordoñez, M. Synthesis of chiral 1,4,2-oxazaphosphepines. Molecules 2015, 20, 13794-13813. [CrossRef]

34. Palmieri, G.; Cimarelli, C.; Volpini, E. Ready $N$-alkylation of enantiopure aminophenols: Synthesis of tertiary aminophenols. Tetrahedron 2001, 57, 6089-6096. [CrossRef] 
35. Palmieri, G. Synthesis of enantiopure $o$-hidroxybenzylamines by stereoselective reduction of 2-imidoylphenols: Application in the catalytic enantioselective addition of diethylzinc to aldehydes. Eur. J. Org. Chem. 1999, 805-811. [CrossRef]

36. Andreu, R.; Ronda, J.C. Synthesis of 3,4-Dihydro-2H-1,3-benzoxazines by Condensation of 2-Hydroxyaldehydes and Primary Amines: Application to the Synthesis of Hydroxy-Substituted and Deuterium-Labeled Compounds. Synth. Commun. 2008, 38, 2316-2329. [CrossRef]

37. Aliouane, N.; Helesbeux, J.-J.; Douadi, T.; Khan, M.A.; Bouet, G.; Chafaa, S.; Duval, O. Synthesis of new benzylic di-, tri-, and tetraphosphonic acids as potential chelating agents. Phosphorus Sulfur Silicon Relat. Elem. 2011, 186, 354-364. [CrossRef]

38. Gibadullina, E.M.; Shaekhov, T.R.; Voronina, Y.K.; Pudovik, M.A.; Burilov, A.R. Reactions of [(3,5-Di-tert-butyl4-oxocyclohexa-2,5-dien-1-ylidene)methyl]phosphonates with Phenols. Russ. J. Org. Chem. 2018, 54, 530-536. [CrossRef]

39. Zengwei, L.; Zhaobin, W.; Jianwei, S. Organocatalytic asymmetric nucleophilic addition to o-quinone methides by alcohols. Org. Lett. 2015, 17, 6058-6061. [CrossRef]

40. Malhotra, B.; Gandelman, K.; Sachse, R.; Wood, N.; Michel, M.C. The design and development of fesoterodine as a prodrug of 5-hydroxymethyl tolterodine (5-HMT), the active metabolite of tolterodine. Curr. Med. Chem. 2009, 16, 4481-4489. [CrossRef]

41. Greene, M.A.; Yonova, I.M.; Williams, F.J.; Jarvo, E.R. Traceless directing group for stereospecific nickel-catalyzed alkyl-alkyl cross-coupling reactions. Org. Lett. 2012, 14, 4293-4296. [CrossRef]

42. Kawaguchi, A.W.; Sudo, A.; Endo, T. Polymerization-depolymerization system sased on reversible addition-dissociation reaction of 1,3-benzoxazines with thiol. ACS Macro Lett. 2013, 2, 1-4. [CrossRef]

43. Metlushka, K.E.; Kashemirov, B.A.; Zheltukhin, V.F.; Sadkova, D.N.; Buchner, B.; Hess, C.; Kataeva, O.N.; McKenna, C.E.; Alfonsov, V.A. 1-( $\alpha$-Aminobenzyl)-2-naphthol: A new chiral auxiliary for the synthesis of enantiopure $\alpha$-aminophosphonic acids. Chem. Eur. J. 2009, 15, 6718-6722. [CrossRef] [PubMed]

Sample Availability: Samples of the compounds are not available from the authors.

(C) 2019 by the authors. Licensee MDPI, Basel, Switzerland. This article is an open access article distributed under the terms and conditions of the Creative Commons Attribution (CC BY) license (http://creativecommons.org/licenses/by/4.0/). 